\title{
Efeito da simulação clínica na retenção do conhecimento de estudantes de enfermagem
}

Effect of clinical simulation on the knowledge retention of nursing students Efecto de la simulación clínica en la retención de conocimientos de estudiantes de enfermería

\author{
Marília Souto de Araújo ${ }^{1}$ ic https://orcid.org/0000-0001-9636-1991 \\ Soraya Maria de Medeiros ${ }^{1}$ io https://orcid.org/0000-0003-2833-9762 \\ Raphael Raniere de Oliveira Costa ${ }^{1}$ id https://orcid.org/0000-0002-2550-4155 \\ Verónica Rita Dias Coutinho² id https://orcid.org/0000-0001-8073-4562 \\ Alessandra Mazzo ${ }^{3}$ id https://orcid.org/0000-0001-5074-8939 \\ Yanna Gomes de Sousa ${ }^{1}$ iD https://orcid.org/0000-0002-0830-5349
}

Araúio MS Momo citar:

SM, Costa RR, Coutinho

VR, Mazzo A, Sousa YG. Efeito da simulação

clínica na retenção do conhecimento de estudantes de enfermagem. Acta Paul Enferm. 2021;34:AAPE000955.

DOI

http://dx.doi.org/10.37689/actaape/2021A0000955

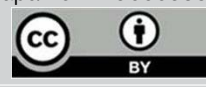

Descritores

Simulação; Emergências; Conhecimento; Estudantes de enfermagem; Atenção Primária à

Saúde

Keywords

Simulation technique; Emergencies; Knowledge: Students, nursing; Primary Health Care

Descriptores

Simulación; Urgencias médicas; Conocimiento; Estudiantes de enfermeira; Atención Primaria de

Salud

Submetido 27 de Abril de 2020

Aceito

23 de Março de 2021

Autor correspondente

Marília Souto de Araújo

E-mail: mariliasdearaujo@yahoo.com.br

\section{Resumo}

Objetivo: Identificar o efeito da simulação clínica no desempenho cognitivo imediato e retido de estudantes do curso técnico de enfermagem acerca da atuação nas emergências na Atenção Primária à Saúde.

Métodos: Estudo de intervenção, do tipo antes e depois, realizado com 46 estudantes do curso técnico em enfermagem. Estes foram alocados em dois grupos: controle e experimental. 0 grupo controle teve acesso a uma aula teórica com demonstração de habilidade. 0 grupo experimental teve acesso a sessão de simulação com quatro cenários. Além da caracterização sociodemográfica, os estudantes responderam a um teste de conhecimento em três momentos, a saber: pré-teste, pós-teste imediato e pós-teste tardio (após 30 dias). Adotou-se um nível de significância de 5\%, através da utilização do teste de Mann-Whitney e de Kruskal Wallis, Resultados: Houve diferença estatística entre as medianas do grupo controle e experimental no pós-teste tardio ( $p$ valor $=0,038$ ), dessa forma, os estudantes do grupo experimental retiveram mais conhecimento que os do grupo controle.

Conclusão: A aprendizagem a partir da simulação clínica conferiu scores e retenção do conhecimento maiores comparados àqueles adquiridos por meio de aula expositiva com demonstração de habilidades.

\section{Abstract}

Objective: To identify the effect of clinical simulation on the immediate and retained cognitive performance of students in a vocational course in nursing about their performance in emergencies in Primary Health Care.

Methods: This is an intervention study, of before and after type, carried out with 46 students of a vocational course in nursing. Students were allocated into two groups: control and experimental. The control group had access to a theoretical class with demonstration of skill. The experimental group had access to a simulation session with four scenarios. In addition to the sociodemographic characterization, students responded to a knowledge test in three moments, namely: pre-test, immediate post-test and late post-test (after 30 days). A significance level of $5 \%$ was adopted, using the Mann-Whitney and Kruskal Wallis tests,

Results: There was a statistical difference between the medians of control and experimental groups in the late post-test ( $p$ value $=0.038$ ), thus students in the experimental group retained more knowledge than those in the control group.

Conclusion: Learning from clinical simulation conferred higher scores and retention of knowledge compared to those acquired through an expository class with demonstration of skills. 


\section{Resumen}

Objetivo: Identificar el efecto de la simulación clínica en el desempeño cognitivo inmediato y retenido de estudiantes de la carrera técnica de enfermería sobre la actuación en emergencias en la Atención Primaria de Salud.

Métodos: Estudio experimental, tipo antes y después, realizado con 46 estudiantes de la carrera técnica de enfermería. Los participantes fueron separados en dos grupos: de control y experimental. El grupo de control tuvo una clase teórica con demostración de habilidades. El grupo experimental tuvo una sesión de simulación con cuatro escenarios. Además de la caracterización sociodemográfica, los estudiantes respondieron una prueba de conocimiento en tres momentos, a saber: pretest, postest inmediato y postest tardío (después de 30 días). El nivel de significación adoptado fue del $5 \%$, mediante la utilización de la prueba de Mann-Whitney y de Kruskal-Wallis.

Resultados: Se observó diferencia estadística entre las medianas del grupo de control y experimental en el postest tardío ( $p$ valor $=0,038)$. De esta forma, los estudiantes del grupo experimental retuvieron más conocimientos que los del grupo de control.

Conclusión: El aprendizaje a partir de la simulación clínica demostró una mayor puntuación y retención de conocimientos comparado con los adquiridos mediante la clase expositiva con demostración de habilidades.

Registro Brasileiro de Ensaios Clínicos (ReBEC): UTN: U1111-1236-9025

\section{Introdução}

A Atenção Primária à Saúde (APS), desde o ano de 2006, através da elaboração da Política Nacional de Atenção às Urgências (PNAU), é considerada porta de entrada para o primeiro atendimento às urgências, na perspectiva de descentralizar a atenção e aumentar a sobrevida dos pacientes. Essa definição se deu considerando, principalmente, a distribuição geográfica das unidades de saúde e a necessidade de rapidez no atendimento. ${ }^{(1)}$

Entretanto, mesmo a PNAU tendo sido promulgada há mais de uma década, ainda observa-se, em alguns contextos, centralidade no nível de atenção terciário na abordagem ao paciente grave, o que traz a necessidade de reflexóes acerca dessa problemática. ${ }^{(1,2)}$

Estudo demonstrou que o conhecimento dos profissionais é fragilizado no âmbito de urgência e emergência; que na APS geralmente os profissionais não se reconhecem como responsáveis por esses acometimentos e que as unidades têm deficiência de estrutura física e material, o que traz piora no prognóstico do paciente e cria a sobrecarga de outros serviços da rede de atendimento. ${ }^{(3)}$

No contexto da enfermagem, diversas pesquisas apontam lacuna no conhecimento e aptidão ${ }^{(4,5)}$ o que geralmente colabora com a falta de resolutividade dos problemas. Muitos profissionais se sentem inseguros e informam que realizaram cuidados relacionados ao tema pela primeira vez, no próprio paciente, sem nem mesmo terem tido a experiência de treinarem em ambientes de laboratório. ${ }^{(6)}$ Dessa maneira, a fragilidade no conhecimento acerca dos protocolos iniciais de atendimento às urgências contribui para o aumento da mortalidade de pacientes, na superlotação de serviços de nível secundário e terciário e nos onerosos gastos em saúde. Com isso, tem-se na educação a estratégia mais eficaz de reduzir essa problemática.

Dentre os profissionais que compóe o corpo da saúde, a enfermagem se destaca em termos quantitativos; os profissionais técnicos em enfermagem (TE) correspondem a $80 \%$ da classe, o que gera a necessidade de reflexóes acerca dos impactos da prática assistencial que vem desempenhando e da formação à que estão sendo expostos. ${ }^{(7)}$ No contexto da APS, essa realidade não difere, os TE representam um significante quantitativo profissional, o que presume a necessidade de verificar o conhecimento e a conduta desses trabalhadores frente às urgências nesse âmbito.

Sabe-se que a escolha correta das tecnologias educacionais e das estratégias de ensino e aprendizagem durante o processo formativo podem determinar o sucesso ou insucesso da aprendizagem, seja no âmbito de formação acadêmica e/ou educação permanente. Ao considerar sua pertinência e aplicabilidade no ensino em saúde, pesquisadores sugerem que profissionais de saúde e instituiçóes de educação e formação utilizem simulaçóes na educação de seus alunos e em capacitaçóes. ${ }^{(8,9)}$

Corroborando essa orientação, estudos têm demonstrado a contribuição da simulação em várias áreas clínicas, bem como no desenvolvimento cognitivo e psicomotor. ${ }^{(8,10)}$ Além disso, sabe-se que a simulação auxilia na consolidação do conhecimento, no desenvolvimento de técnicas, nas habilida- 
des relacionais, no pensamento, na reflexão, assim como no desenvolvimento profissional..$^{(8,11,12)}$

No contexto nacional e internacional, poucos estudos têm se debruçado a estudar a formação do TE e mais precisamente a simulação clínica no ensino médio profissionalizante, mesmo que, no contexto brasileiro, se reconheça fragilidades nessa formação. ${ }^{(13)}$

$\mathrm{Na}$ presente pesquisa, o desempenho cognitivo está relacionado ao conhecimento adquirido, fundamentado através da Pirâmide de Miller. Dessa forma, avalia-se como os estudantes relacionam os conhecimentos prévios com os novos; considera-se que o saber é premissa para que o estudante avance nos outros níveis, tais como o "sabe como", "mostra como" e o "faz".

Nesse contexto, objetivou-se identificar o efeito da simulação clínica no desempenho cognitivo de estudantes do curso técnico de enfermagem acerca da atuação nas emergências na Atenção Primária à Saúde.

\section{Métodos}

Trata-se de um estudo de intervenção, com abordagem quantitativa compreendida em três fases. Foi utilizado o delineamento quase-experimental de grupo controle não-equivalente do tipo pré-teste, pós-teste imediato e pós-teste tardio. $\mathrm{O}$ estudo foi desenvolvido em uma universidade pública federal, entre setembro e novembro de 2019.

Participaram do estudo os estudantes do curso técnico em enfermagem da referida instituição. Ressalta-se que a instituição atua na formação em nível técnico, superior e pós-graduação. Através de um processo de amostragem náo-probabilística por conveniência, participaram do presente estudo 46 estudantes.

Realizou-se a divulgação do curso nas cinco turmas do curso técnico em enfermagem. Foram incluídos os estudantes regularmente matriculados na instituição, que estiveram presentes durante a aplicação dos instrumentos da pesquisa e das intervenções. Os faltosos nas intervenções e aqueles que tinham experiência profissional na área da saúde foram excluídos do estudo.
Posteriormente, os interessados no curso preencheram manualmente a ficha de inscrição disponibilizada. A ficha continha dados referentes a nome, telefone, e-mail, e algumas informações preliminares acerca da proximidade do estudante com a temática abordada.

Inicialmente, foram apresentados os objetivos da pesquisa aos estudantes. Mediante a concessão individual, foi aplicado um instrumento de caracterização sociodemográfica, com as seguintes variáveis: sexo, idade e "estudou Suporte Básico de Vida (SBV) anteriormente". Posteriormente, o arrolamento dos sujeitos foi realizado, sendo os estudantes distribuídos, através de sorteio, no grupo controle ou no experimental. A figura 1 esquematiza o percurso metodológico que foi seguido.

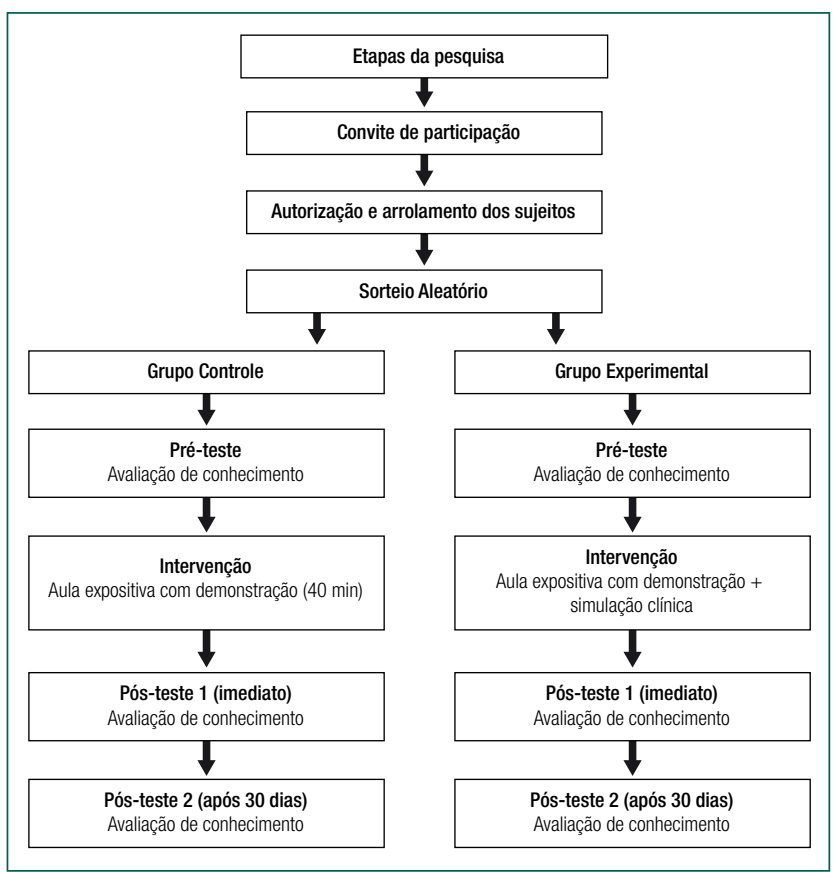

Figura 1. Fluxograma das etapas metodológicas da pesquisa

A avaliação de conhecimento possuía 10 questôes de múltipla escolha referentes aos conteúdos de suporte básico de vida. Foi adotado para cada questão o valor de 1 ponto, obtendo o máximo de acertos o estudante que obtiver 10 pontos. Além disso, para cada questáo, o estudante assinalou a opção que define o nível de certeza que ele possuía no momento da resolução da questáo.

A avaliação de conhecimento foi construída pelos pesquisadores após realização de levantamento da litera- 
tura e dos protocolos internacionais da American Heart Association (AHA) sobre os SBV mais atualizados até a data de coleta de dados. Após essa etapa, procedeu-se a sua aplicação, através de teste piloto, para realizar ajustes de semântica e clareza de conteúdo. Não foram apontadas sugestões de modificaçóes. Posteriormente, foi aplicada em três momentos: pré-teste e pós-teste imediato e pós-teste tardio (após 30 dias da intervenção).

O grupo controle participou do curso teórico-prático de Suporte Básico de Vida com ênfase na Atenção Primária à Saúde, com estratégia metodológica de aula expositiva com demonstração de habilidades. Já o grupo experimental participou do mesmo curso, porém, com a estratégia de aula expositiva com demonstração de habilidades e simulação clínica.

Para a realização da simulação, foi seguido o modelo conceitual de Simulação NLN/Jeffries para o ensino de enfermagem, proposto por Jeffries. ${ }^{(14)}$

Os cenários de simulação foram construídos e executados previamente, a fim de averiguar possíveis falhas e realizar adaptaçóes dos desenhos. Cada cenário possuiu um objetivo de aprendizagem relacionado a vivência de uma situação de parada cardiorrespiratória no adulto, na gestante e uma situação de engasgo, precedida de PCR no âmbito da APS. Todos os cenários continham os seguintes aspectos: 1) Objetivo geral e específico; 2) Competências e habilidades; 3) Materiais; 4) Descrição do caso; 5) Questôes norteadoras para o debriefing; 5) Checklist para acompanhamento de performace prática. Ressalta-se que o checklist foi criado com único fim de auxiliar os pesquisadores na condução do debriefing; os resultados da peformace prática dos estudantes não foram consideradas para o estudo.

Um teste piloto foi executado, além de prévia capacitação da equipe para a coleta de dados. Para o desenvolvimento das simulaçóes, utilizou-se manequins de baixa fidelidade, que são simuladores estáticos, e paciente simulado, que são atores treinados para atuar e reproduzir comportamentos de usuários em diversas situaçóes e estabelecimentos de assistência à saúde. ${ }^{(11)}$ Dessa forma, cada cenário foi conduzido por um facilitador que, logo após o encerramento, realizou o debriefing.

Após as testagens dos cenários e dos instrumentos, o curso foi iniciado com a realizaçáo de quatro cenários de simulação, cada um com duração de 30 minutos, com a seguinte programação: 5 minutos para o briefing, 5 minutos para a ação e 20 minutos para o debriefing.

Desde o primeiro momento - aula expositiva com demonstraçáo de habilidades - foram observadas perdas de sujeitos do estudo. Dos 86 inscritos, 60 compareceram à aula teórica. Os alunos foram redivididos por sorteio, a fim de que o grupo controle (30) e o experimental (30) ficassem homogêneos. No momento de coletar o pós-teste tardio, houve outras perdas (14). Estas podem ser visualizadas no fluxograma na figura 2 .

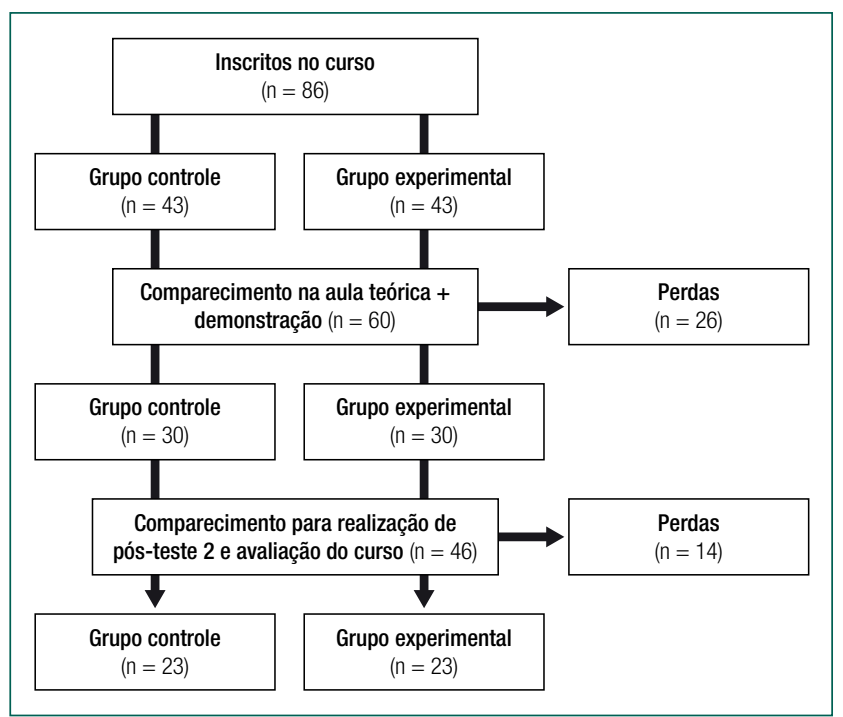

Figura 2. Fluxograma da amostra inicial, final e perdas do estudo

\section{Análise e tratamento dos dados}

As variáveis utilizadas no estudo são: sexo, idade, índice de rendimento escolar e conhecimento. Para as variáveis categóricas foi utilizada a frequência relativa e absoluta, e para as variáveis escalares, medidas de tendência central. Os dados coletados foram inseridos em uma planilha do Programa Estatístico Statistical Package for the Social Sciences (SPSS) versão 20.0, sendo realizada análise descritiva e inferencial. Dessa forma, na estatística inferencial, para determinação das medidas de efeito, significância estatística e intervalos de confiança foram aplicados os testes de Mann-Whitney para as comparações entre os grupos, considerando que as duas amostras independentes são de caráter não normal, resultando na necessidade de uso de testes não-paramétricos, e para as associaçóes 
utilizando a faixa de idade e para a análise intragrupos foi utilizado o teste de Kruskal Wallis. Considerou-se significativos os resultados com $\mathrm{p} \leq 0,05$.

$\mathrm{O}$ estudo levou em consideração a garantia dos princípios éticos e legais que regem a pesquisa em seres humanos, preconizados na Resolução no 466/2012 do Conselho Nacional de Saúde (CNS) do Ministério da Saúde, sendo submetido e aprovado junto ao Comitê de Ética em Pesquisa sob o protocolo no 3.305.558 e CAAE $n^{\circ}$ 09905819.0.0000.5537. Após aprovação, o estudo foi cadastrado na plataforma de Registro Brasileiro de Ensaios Clínicos, sob protocolo REQ: 8480, de número do UTN: U1111-1236-9025.

É importante destacar que, após a finalização da pesquisa, os pesquisadores convidaram os estudantes do grupo controle para uma sessão de experiências clínicas simuladas. $\mathrm{Na}$ oportunidade, os estudantes puderam vivenciar os cenários simulados anteriormente trabalhados somente entre os estudantes do grupo experimental.

\section{Resultados}

Participaram do presente estudo 46 estudantes, predominantemente do sexo feminino $(69,6 \%)$ e na faixa etária compreendida entre 16 e 25 anos (63\%) e que não haviam estudado suporte básico de vida na grade regular do curso técnico (76,9\%). Através do teste qui-quadrado, verificou-se a homogeneidade dos grupos ( $p$-value $\geq 0,05)$ (Tabela 1$)$.

Tabela 1. Caracterização sociodemográfica da amostra

\begin{tabular}{lcc|c}
\hline Variáveis & $\begin{array}{c}\text { Controle } \\
\mathbf{n}(\%)\end{array}$ & $\begin{array}{c}\text { Experimental } \\
\mathbf{n}(\%)\end{array}$ & $\begin{array}{c}\text { Total } \\
\mathbf{n}(\%)\end{array}$ \\
\hline Sexo & & & \\
$\quad$ Feminino & $19(59,4)$ & $13(40,6)$ & $32(69,6)$ \\
$\quad$ Masculino & $4(28,6)$ & $10(71,4)$ & $14(30,4)$ \\
Total & 23 & 23 & $46(100,0)$ \\
Faixa etária & & & \\
$\quad 16$ a 25 anos & $13(44,8)$ & $16(55,2)$ & $29(63,0)$ \\
26 a 35 anos & $5(62,5)$ & $3(37,5)$ & $8(17,4)$ \\
36 a 45 anos & $3(50,0)$ & $3(50,0)$ & $6(13,0)$ \\
$\quad$ A partir de 46 anos & $2(66,7)$ & $1(33,3)$ & $3(6,5)$ \\
Total & & & $46(100,0)$ \\
Estudou SBV no curso & & & \\
$\quad$ Não & & $16(47,1)$ & $34(73,9)$ \\
$\quad$ Sim & $18(52,9)$ & $7(58,3)$ & $12(26,1)$ \\
Total & $5(41,7)$ & 23 & $46(100,0)$ \\
\hline
\end{tabular}

n - frequência absoluta; \% - frequência relativa; SBV - Suporte Básico de Vida
Para identificar e comparar os scores de conhecimento acerca do ensino de suporte básico de vida no contexto da Atenção Primária à Saúde entre os grupos controle e experimental, foi aplicado um instrumento referente à avaliação de conhecimento em três momentos: Pré-teste (antes da intervenção), Pós-teste 1 (imediatamente após a intervenção) e pós-teste 2 (após 30 dias da intervenção).

A tabela 2 apresenta os resultados do teste de Mann-whitney realizado para testar se houve diferenças entre as medianas do grupo controle e experimental, segundo a avaliaçáo de conhecimento nos três momentos. Obteve-se ao nível de significância $5 \%$, a rejeição da hipótese de que as medianas dos grupos são iguais, ou seja, há evidências de que existe diferença entre a mediana do grupo controle e do experimental no pós-teste $2(\mathrm{p}<0,05)$.

Quanto ao pré-teste e ao pós-teste 1, os resultados não revelaram diferença estatística entre as medianas dos grupos; os estudantes do grupo experimental retiveram mais conhecimento quando comparados aos do grupo controle.

Tabela 2. Comparação do desempenho cognitivo entre o grupo controle e experimental, no pré-teste, pós-teste 1 e pós-teste 2

\begin{tabular}{ll|c|c|c|c|c|c|}
\hline Momento & Grupo & Média & Mediana & DP & Mín & Máx & $\boldsymbol{p}_{\text {-value }}$ \\
\hline Pré & Controle & 3,7 & 4,0 & 2,1 & 1,0 & 8,0 & 0,470 \\
& Experimental & 4,2 & 4,0 & 2,0 & 1,0 & 8,0 & \\
Pós 1 & Controle & 7,7 & 8,0 & 1,3 & 5,0 & 10,0 & 0,103 \\
& Experimental & 7,1 & 7,0 & 1,4 & 4,0 & 9,0 & \\
Pós 2 & Controle & 5,9 & 6,0 & 1,9 & 1,0 & 9,0 & 0,038 \\
& Experimental & 6,8 & 7,0 & 1,3 & 3,0 & 8,0 & \\
\hline
\end{tabular}

DP - Desvio-padrão; Mín - mínimo; Máx - máximo; * Teste de Mann-Whitney

Além da comparação entre o grupo controle e experimental, foi realizada a análise intragrupo através do teste de Kruskal Wallis e de Dunn - com o objetivo de verificar se houve diferença estatística entre as medianas dos três momentos (pré-teste, pós-teste 1 e pós-teste 2) dentro de cada grupo. Dessa maneira, evidenciou-se significância estatística nos três momentos do grupo controle, considerando que no pré-teste as medianas eram baixas, no pós-teste 1 aumentaram significativamente e no pós-teste 2, reduziram. No grupo experimental, houve significância no pré-teste e no pós-teste 1 , não havendo no pós-teste 2 ; no pré-teste as medianas eram baixas, no pós-teste 1 aumentaram consideravelmente e no pós-teste 2 permaneceram altas 
Tabela 3. Associação da avaliação de conhecimento com as variáveis "estudado Suporte Básico de Vida anteriormente" e "faixa etária" no pré-teste, pós-teste imediato e pós-teste tardio entre grupo controle e experimental

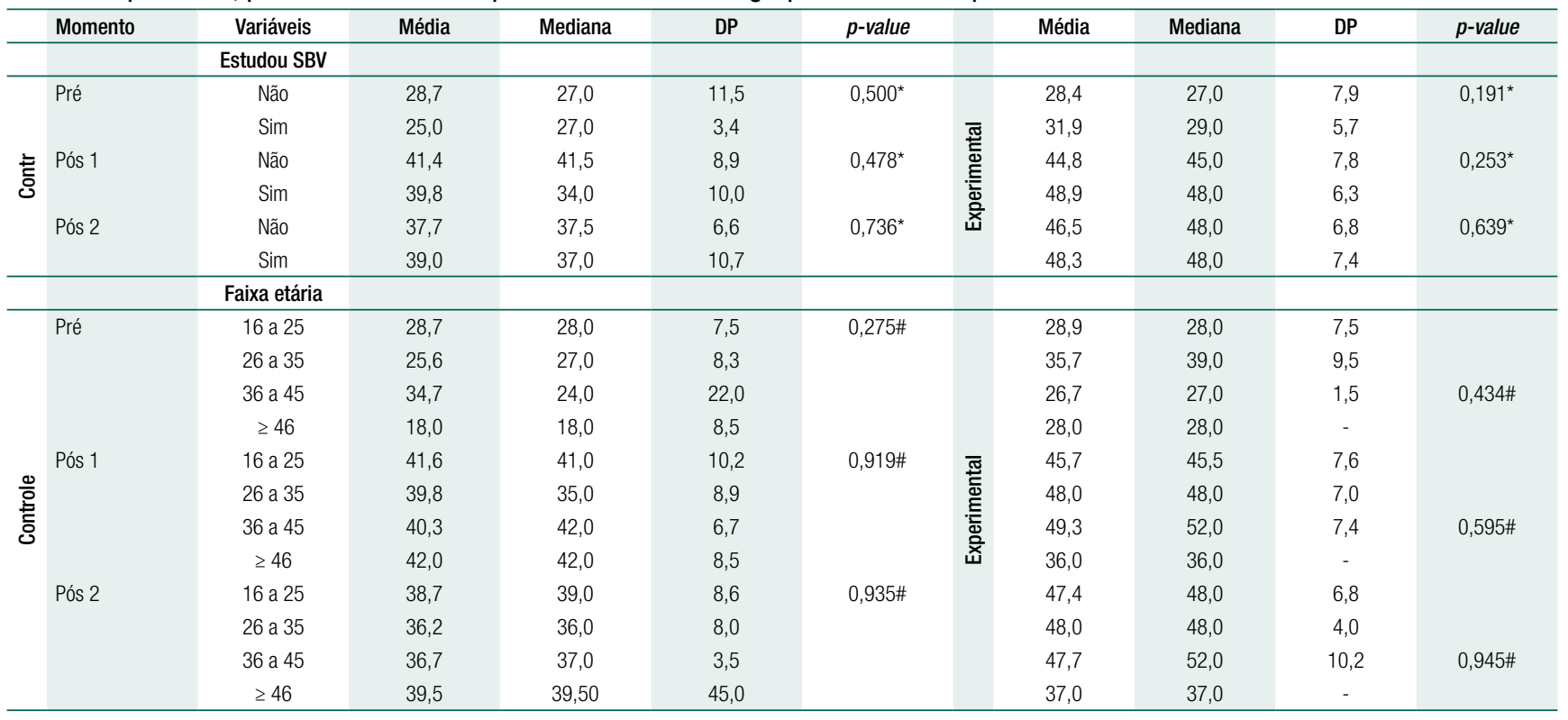

SBV - Suporte Básico de Vida; DP - desvio padrão; *Teste de Mann-Whitney; \# Teste de Kruskal Wallis

$(\mathrm{p}>0,05)$. Na tabela 3 tem-se demonstrado a análise, através do Teste de Mann-Whitney e Kruskal Wallis, da possível relação entre a aquisição e retenção de conhecimento com as seguintes variáveis: estudou Suporte Básico de Vida (SBV) anteriormente e a faixa etária. Os resultados apontaram para a não relação dessas variáveis $(\mathrm{p}>0,05)$.

\section{Discussão}

Na presente pesquisa, o desempenho cognitivo dos estudantes foi verificado através da avaliação de conhecimento, aplicada em três momentos: pré-teste, pós-teste imediato e após 30 dias da realização da intervenção. O conhecimento dos estudantes examinados, tanto do grupo controle quanto do experimental, mostraram-se semelhantes no pré-teste e no pós-teste imediato.

No pré-teste, ambos os grupos apresentaram médias baixas (grupo controle: 3,7 pontos; e grupo experimental: 4,2 pontos). Após as intervençóes nos dois grupos, as médias aumentaram significativamente.

Resultados de um estudo ${ }^{(15)}$ corroboraram com aqueles aqui apresentados onde em uma capacitação em ressuscitação cardiopulmonar (RCP) e simulação clínica para alunos do ensino médio de uma escola pública e outra privada observou-se, no aprendizado dos estudantes, uma melhora significativa tanto no pós-teste imediato quanto no tardio. ${ }^{(15)}$

Ademais, o presente trabalho ainda identificou que houve diferença estatística entre as medianas do grupo controle e do experimental no pós-teste 2 $(\mathrm{p}<0,05)$. Com isso, tem-se que os estudantes do grupo experimental sustentaram, após 30 dias, melhora significativa de conhecimento. Tais resultados ratificam aqueles encontrados em diversos estudos que avaliaram a retenção de conhecimento de estudantes através do uso da simulação, apontando que estudantes que fazem utilização dessa estratégia de ensino adquirem um conhecimento mais duradouro. ${ }^{(8,16-18)}$

Nessa esteira, uma pesquisa que analisou a retenção de conhecimento de estudantes de medicina no contexto da ressuscitação cardiopulmonar após 42 meses da intervenção educativa, evidenciou que os alunos que tiveram a simulação como estratégia de ensino retiveram mais conhecimento do que os que não tiveram. ${ }^{(16)}$ Em contraponto a esse estudo, uma pesquisa que avaliou a retenção de conhecimento através de um curso de Suporte Básico de Vida baseado em simulação resultou em aprendizagem prática e teórica sobre ressuscitação cardiopulmonar, entretanto, o efeito de retenção positiva não se manteve após um ano e cinco meses, exceto em participantes 
que repetiram o treinamento nesse período, indicando que a retenção de conteúdo em longo prazo exige mais oportunidades de treino ou prática. ${ }^{(19)}$

Ensaio clínico controlado que avaliou a eficácia do uso da simulaçáo no processo de ensino e aprendizagem em relação ao ensino tradicional, demonstrou que os dois grupos apresentaram melhora do conhecimento, entretanto, os estudantes do grupo experimental retiveram mais conhecimento que os do controle. ${ }^{(20)}$

Destaca-se aqui a diferença no desempenho cognitivo que a utilização da simulação clínica promove, apresentando potenciais impactos positivos na aprendizagem de estudantes. Tem-se claro, pois, o grande potencial da simulação para tratar de problemas relacionados ao desenvolvimento de conhecimentos e competência dos profissionais, na medida em que supera o processo de recepção passiva centrado na transmissão de conteúdos, presente na maioria dos currículos. ${ }^{(21)}$ Através da pirâmide de Miller, identifica-se que quando um currículo é predominantemente composto por sequências de ensino onde predominam a disposição de conteúdos, tem-se negligenciado o "saber como" e o "mostrar como", comprometendo consideravelmente o processo de ensino e aprendizagem dos estudantes. ${ }^{(22)}$

Especificamente, tratando-se da retenção de conhecimento adotada nesse estudo, através de uma avaliação após 30 dias da intervenção, tem-se que os resultados corroboraram os encontrados nos estudos discutidos anteriormente, onde resta manifesto que as experiências simuladas contribuem na aquisição de diferentes competências e promovem maior aproveitamento e, consequentemente, maior retenção do conhecimento.

Reflete-se ainda sobre a simulação clínica enquanto estratégia de ensino eficaz no processo de formação técnica em enfermagem no Brasil; foi verificado no pré-teste um baixo nível de conhecimento acerca do assunto de Suporte Básico de Vida voltado para as emergências na Atenção primária à Saúde, onde tanto o grupo controle quanto o experimental obtiveram uma média de 3,9 pontos (máximo $=10$ pontos).

Autores $^{(23)}$ apontam que os níveis de conhecimentos dos técnicos de enfermagem são insuficientes no que se refere a assuntos inerentes à prática assistencial. Com isso, reflete-se sobre o impacto dessa la- cuna para a assistência de enfermagem, considerando que os profissionais técnicos em enfermagem representam a maior categoria profissional da saúde, dessa forma, presume-se que a assistência oferecida por eles impactam consubstancialmente na saúde brasileira. ${ }^{(7)}$

Importante, nesse momento, refletir sobre a formação dessa categoria, principalmente no que se refere à constituição do corpo docente, as metodologias de ensino utilizadas, distribuição de carga-horária e equiparação do currículo às normativas ministeriais.

Existe uma lacuna científica acerca de estudos que utilizam estudantes ou profissionais técnicos em enfermagem como população e a formação deles como objeto de estudo. Assim como podemos apresentar como fatores limitantes desse estudo, as experiências posteriores dos estudantes as práticas simuladas. Entretanto, os resultados apresentados na presente pesquisa apontam para um conhecimento primariamente deficiente e que após ensino com utilização de simulação clínica, aumenta consubstancialmente os níveis de conhecimento desses estudantes não só de forma pontual como tardia.

Dessa forma, o estudo apresenta resultados de aumento de conhecimento de estudos, que presume uma melhoria da qualificação de futuros profissionais a partir da utilização da simulação clínica, que, em sua gênese estrutural, apresenta - quando utilizada de forma efetiva - contribuiçóes que transpassa as salas de aula, o conhecimento pontual e as avaliaçóes com fins somativos.

Os autores do presente estudo recomendam a utilização da simulação clínica enquanto estratégia de ensino a ser utilizada no ensino médio profissionalizante em enfermagem, com vistas a aumentar os níveis de conhecimento e de retenção da aprendizagem. Como limitação do estudo, tem-se a impossibilidade de avaliar uma retenção de conhecimento mais prolongada, o uso de questionários de avaliação de conhecimento não validados entre outros pares, para além da equipe da pesquisa, e o número amostral reduzido em consequência da perda amostral.

\section{Conclusão}

Os estudantes que tiveram a oportunidade de ter um aprendizado a partir da utilização da metodologia de simulação clínica, retiveram um melhor desempenho 
cognitivo em longo prazo (30 dias) quando comparados àqueles que foram submetidos a uma estratégia de ensino tradicional. Além disso, evidenciou-se, no contexto pesquisado, que estudantes mais jovens, do sexo feminino e entre 16 e 25 anos, aprendem mais em comparação aos demais. Dessa forma, estimula-se a utilização de simulação clínica no ensino em enfermagem, considerando-se seu efeito positivo no desempenho cognitivo - a curto e longo prazo, o que tende a colaborar com aspectos que transpassam a formação profissional pessoal, tendo-se em conta os impactos sociais de uma assistência à saúde de qualidade, eficaz e segura.

\section{Agradecimentos}

O presente trabalho foi realizado com apoio do Conselho Nacional de Desenvolvimento Científico e Tecnológico (CNPq) - Brasil - Código de Financiamento 001, ao conceder bolsa de mestrado à Marília Souto de Araújo.

\section{Colaborações}

Araújo MS, Medeiros SM, Costa RRO, Coutinho VRD, Mazzo A e Sousa YG contribuíram com a concepção, redação, revisão crítica relevante do conteúdo intelectual e aprovação da versão final do artigo.

\section{Referências}

1. Faria TL, Nascimento DM, Farias Filho MC, Nunes SF. National policy of urgency and emergency under the Federal Coordination in Pará Municipalities, Brazil. Saude Soc. 2017;26(3):726-37.

2. O'Dwyer G, Konder MT, Reciputti LP, Macedo C, Lopes MG. 0 processo de implantação do Serviço de Atendimento Móvel de Urgência no Brasil: estratégias de ação e dimensões estruturais. Cad Saúde Pública. 2017;33(7):e00043716.

3. Farias DC, Celino SD, Peixoto JB, Barbosa ML, Costa GM. Acolhimento e Resolubilidade das Urgências na Estratégia Saúde da Família. Rev Bras Educ Med. 2015;39(1):79-87.

4. Silva DP, Santos IM, Torres PM, Dantas HL, Moreira RT. The knowledge of undergraduate students in first aid: an integrative review. Health Society Port J. 2019;4(1):105561.

5. Silva KR, Araújo SA, Almeida WS, Pereira IV, Carvalho EA, Abreu MN. Parada cardiorrespiratória e o suporte Básico de vida no ambiente pré-hospitalar: 0 saber acadêmico. Rev Saúde (Santa Maria). 2017;43(1):53-9.
6. Almeida RG, Mazzo A, Martins JC, Jorge BM, Souza Júnior VD, Mendes IA. Self-confidence in the care of critically ill patients: before and after a simulated intervention. Rev Bras Enferm. 2019;72(6):161823.

7. Araújo MS, Medeiros SM, Costa EO, Oliveira JS, Costa RR, Sousa YG. Analysis of the guiding rules of the nurse technician's practice in Brazil. Rev Bras Enferm. 2020;73(3):e20180322.

8. Costa RR, Medeiros SM, Coutinho VR, Mazzo A, Araújo MS. Satisfaction and self-confidence in the learning of nursing students: randomized clinical trial. Esc Anna Nery. 2020;24(1):e20190094.

9. Ribeiro VS, Garbuio DC, Zamariolli CM, Eduardo AH, Carvalho EC. Clinical simulation and training for Advanced Nursing Practices: an integrative review. Acta Paul Enferm. 2018;31(6):659-66.

10. Góes FS, Aredes ND, Hara CY, Fonseca LM, Campbell SH. Simulation with standardized patients: nursing student's communication skills in health. Rev Rene. 2017;18(3):383-9.

11. Costa RR, Medeiros SM, Vitor AF, Lira AL, Martins JC, Araújo MS. Types and purposes of the simulation in undergraduate nursing education: integrative literature review. Rev Baiana Enferm. 2016;30(3):1-11.

12. Jerônimo IR, Campos JF, Peixoto MA, Brandão MA. Use of clinical simulation to improve diagnostic reasoning in nursing. Esc Anna Nery. 2018;22(3):e20170442.

13. Wermelinger MC, Boanafina $A$, Machado $M H$, Vieira $M$, Ximenes Neto FR, Lacerda WF. Nursing technician training: qualification profile. Cienc Saude Coletiva. 2020;25(1):67-78.

14. Jeffries PR. Simulation in nursing education: from conceptualization to evaluation. New York: National League for Nursing; 2012.

15. Boaventura AP, Miyadahira AM. Programa de capacitação em ressuscitação cardiorrespiratória com uso do desfibrilador externo automático em uma universidade. Rev Gaúcha Enferm. 2012;33(1):1914.

16. Saad R. Retenção de conhecimentos e habilidades após treinamento de ressuscitação cardiopulmonar em alunos de uma faculdade de medicina [tese]. São Paulo: Universidade de São Paulo; 2018.

17. Nascimento MS, Magro MC. Realistic simulation: method of improving knowledge and self-confidence of nursing students in the administration of medication. Rev Min Enferm. 2018;22:e-1094.

18. Costa RR, Medeiros SM, Martins JC Coutinho VR, Araújo MS. Eficácia da simulação no ensino de imunização em enfermagem: ensaio clínico randomizado. Rev Lat Am Enfermagem. 2020;28:e3305.

19. Lima MN, Gaspar FD, Mauro TG, Arruda MA, Abbad GS. Retenção da aprendizagem após treinamento em Suporte Básico de Vida com uso de simulação de baixa fidelidade em uma unidade hospitalar odontológica. Sci Med. 2018;28(1):ID29410.

20. Oliveira KM. Implementação da simulação realística como método de melhoria da segurança do paciente: ensaio controlado [dissertação]. Brasília (DF): Universidade de Brasília; 2017.

21. Mesquita HC, Santana BS, Magro MC. Effect of realistic simulation combined to theory on selfconfidence and satisfaction of nursing professionals. Esc Anna Nery. 2019;23(1):e20180270.

22. Costa RR, Medeiros SM, Martins JC, Coutinho VR. Simulation in training nurses: reflections and justifications based on bioethics and human rights approaches. Acta Bioeth. 2018;24(1):31-8.

23. Miyazaki MY, Caliri MH, Santos CB. Knowledge on pressure ulcer prevention among nursing professionals. Rev Lat Am Enfermagem. 2010;18(6):1203-11. 